\title{
Significado del consumo de drogas para las adolescentes de la calle, en la ciudad de Valencia, Venezuela
}

\author{
Roberto Alvarado-Chacón ${ }^{1}$ \\ Marta Angélica lossi Silva² \\ Francisco Guzmán-Facundo ${ }^{3}$ \\ Ronald Igor Serrano-Cazorla ${ }^{4}$ \\ Alexander Bencomo 5
}

Se trata de un estudio descriptivo de enfoque cualitativo con el objetivo de revelar el significado del consumo de drogas en las adolescentes de la calle, condición que las convierte en problema social, por lo que se busca obtener una aproximación teórica para comprender este fenómeno. Siete (7) adolescentes femeninas del paseo Cabriales en la ciudad de Valencia, en Venezuela fueron entrevistadas. Para el análisis de los datos se utilizó el recurso metodológico de la Teoría fundamentada (Grand Theory) y la visión del Interaccionismo simbólico y de ahí surgieron las siguientes categorías: la búsqueda de no ser la buena, la reafirmación del rol femenino, el consumo como experiencia, la mirada de los otros a la adolescente que consume drogas, y la adicta como cuidadora. Finalmente, concluimos que el uso de drogas en la calle para las adolescentes del sexo femenino es una forma de protección, sobrevivencia e inclusión en los grupos.

Descriptores: Adolescente; Jóvenes sin Hogar; Drogas Ilícitas.

\footnotetext{
${ }^{1}$ Licenciado en Enfermería, Magíster en Enfermería Salud Reproductiva, Docente Agregado, Escuela Enfermería, Universidad de Carabobo, Valencia, Venezuela. E-mail: rvarado3@hotmail.com.

2 Enfermera, Doctora en Enfermería. Profesor Doctor, Escola de Enfermagem de Ribeirão Preto, Universidade de São Paulo, Centro Colaborador de la OMS para el Desarrollo de la Investigación en Enfermería, SP, Brasil. E-mail: maiossi@eerp.usp.br.

${ }^{3}$ Doctor en Enfermería. Profesor de tiempo Completo Titular A, Universidad Autonoma de Nuevo León, Nuevo León, México. E-mail: pako2001@hotmail.com.

${ }^{4}$ Licenciado en Enfermería, Licenciado en Psicología, Magíster en Enfermería. Docente Asistente, Escuela de Psicología, Universidad Arturo Michelena, San Diego, Venezuela. E-mail: ronserra91@hotmail.com.

${ }^{5}$ Magíster en Enfermería, Docente Instructor, Escuela Enfermería, Universidad de Carabobo, Valencia, Venezuela. E-mail: albencomo@hotmail.com.
}

Correspondencia:

Marta Angélica Iossi Silva

Universidade de São Paulo. Escola de Enfermagem de Ribeirão Preto

Departamento Materno-Infantil e Saúde Pública

Av. dos Bandeirantes, 3900

Bairro: Monte Alegre

CEP: 14040-902 Ribeirão Preto, SP, Brasil

E-mail: maiossi@eerp.usp.br 


\section{Significado do uso de drogas para adolescentes de rua, Valencia, Venezuela}

Trata-se de estudo descritivo de abordagem qualitativa, cujo objetivo foi revelar o significado do uso de drogas entre adolescentes que vivem nas ruas, condição que se torna um problema social, de modo que a abordagem teórica busca compreender esse fenômeno. Sete (7) adolescentes do sexo feminino, em Valência, Venezuela, foram entrevistadas. Para análise dos dados, utilizou-se como recurso a metodologia da Grounded Theory e a visão do interacionismo simbólico, de onde emergiram as seguintes categorias: a busca de não ser bom, reafirmação do papel das mulheres, consumo como experiência, o olhar dos outros para a adolescente que usa drogas e o toxicodependente como cuidador. Por fim, conclui-se que o uso de drogas na rua, para adolescentes do sexo feminino, é uma forma de proteção, sobrevivência e inclusão grupal.

Descritores: Adolescente; Menores de Rua; Drogas Ilícitas.

\section{Meaning of Drugs Use For Adolescents living in the Streets, Valencia, Venezuela}

This descriptive study with a qualitative approach aims to reveal the meaning of drugs use among adolescents living in the street, a condition that turns them into a social problem, so that theoretical approach seeks to understand the phenomenon. Seven (7) female adolescents living on the Cabriales walk in Valencia, Venezuela were interviewed. Grounded Theory and symbolic interactionism were used for data processing. Thus, the following categories were noticed: The search not to be good, Reaffirmation of the role of women, Consumption as experience, The gaze of others to the adolescent who uses drugs and The addict as a caregiver. Finally, we found that the use of drugs on the street for girls is a form of protection, survival and inclusion in the group.

Descriptors: Adolescent; Homeless Youth; Street Drugs.

\section{Introducción}

El consumo de drogas en el mundo es una conducta remota, ya que desde nuestros ancestros estas substancias han sido utilizadas como medios para provocar alucinaciones y satisfacción, como formas de alejarse de la realidad y obtener placer. Sin embargo, en los últimos años se ha hecho hincapié en los efectos negativos que estas producen en el organismo, lo que induce a investigar, en los grupos vulnerables, a este flagelo(1).

Dentro de estos grupos, tradicionalmente, se cree que están expuestos los jóvenes masculinos, como sujetos inseguros y que se sienten poco felices, mientras que el grupo de mujeres pareciera comportarse como ajeno al fenómeno; sin embargo, en la actualidad, en las ciudades de nuestro país cada vez se visualizan jóvenes del sexo femenino deambulando por las calles y específicamente en la ciudad de Valencia, situación que no corresponde a la figura que ha desempeñado la mujer en la sociedad, ya que la mujer se ha comportado por años como jefe de familia, asumiendo el rol central del control y mantenimiento de ese núcleo, incluso en aquellos hogares donde el hombre está presente, ella se encarga de la casa, de los quehaceres domésticos, de la imposición de normas, es la que muchas veces busca el sustento para la alimentación, manutención, educación, vestimenta y la recreación de los hijos, en definitiva se percibe a la mujer como la que trabaja sin prejuicios y se interesa por el avance y movilidad de su grupo familiar.

Acercarnos a este fenómeno surgió de la experiencia y vivencia social que nos ha permitido ver como se encuentran las adolescentes consumidoras de drogas, que viven en las calles de las Parroquia San José y San Blas en el Municipio Valencia, principalmente en las riberas del río Cabriales, zona emblemática de la delincuencia regional: el Paseo Cabriales; lo que nos llevó 
a reflexionar sobre las circunstancias que rodean este consumo, que está ocurriendo en las calles transitadas por muchachos y muchachas despersonalizados, viviendo en la calle, actuando en roles inapropiados, confrontando a las normas y actividades sociales.

Esta vivencia unida al hecho de que el consumo de drogas ilícitas en Venezuela, así como en la mayoría de los países del mundo, se ha convertido en un problema de salud pública, por el incremento de sus indicadores de morbimortalidad, que en el año 2002 fue el segundo país de América del Sur con mayor prevalencia de consumo de marihuana $(3,3 \%)$ y anfetaminas $(0,6 \%)$, y el tercer país de América del Sur con mayor consumo de cocaína $(1,1 \%)^{(2)}$. En este sentido en Venezuela, se destaca que $19.16 \%$ de las personas que asisten a centros de tratamiento son adolescentes de 15 a 19 años y 2,2\% son menores entre 8 y 14 años, de estos el $12,5 \%$ son de sexo femenino. La droga ilícita de inicio del consumo de mayor frecuencia es la marihuana, seguida por cocaína, bazuco e inhalantes. Un dato que llama la atención es que el $48,3 \%$ de las personas consumidoras de drogas, se inició entre los 10 y 14 años de edad y el $45,1 \%$ entre los 15 y 19 años de edad(3)

En relación a los valores mencionados anteriormente se refleja que existe un grupo de mayor vulnerabilidad que podría pasar a formar parte de esta estadística, representado por la adolescencia, la cual puede ser considerada como un período saludable en el ciclo vital y también una fase crucial en la vida de los individuos en términos de formación de hábitos de conducta y de modelos de socialización, transformando toda la segunda década de la vida en un período de transición.

Es una etapa en que las influencias externas adquieren importancia progresiva; quizás, la mayor independencia de esta fase, cuando es comparada con la niñez, hace que el adolescente se torna más vulnerable en el contexto en que se desenvuelve de forma diferente; siendo que el contexto influencia su salud en su desarrollo físico y psicosocial(4)

Cabe agregar que informes de los procedimientos de incautaciones realizados por los organismos del estados en lo que va del primer semestre del año 2008, dentro de estas cifras se encuentra el estado de Carabobo, prevaleció el género masculino con 2.852 detenidos por el delito de drogas mientras que las detenciones en mujeres fueron 431 en el ámbito nacional(3).

Estos hallazgos permiten afirmar que existe una sub-población que en un momento determinado pueden pasar a formar parte y por consiguiente estar expuestas a conductas vulnerables, tales como los y las adolescentes de la calle, estos grupos no difieren, en esencia de los adolescentes que viven con su familia y frecuentan la escuela, en lo que se refiere a las características universales propias de su desarrollo, sin embargo ellos tienen experiencias muy particulares que los diferencian y transformándolos en un grupo de mayor vulnerabilidad( ${ }^{(5)}$

Por esa razón consideramos de interés aproximarnos al significado del consumo de drogas para las adolescentes de la calle en Valencia-Venezuela, desde una perspectiva menos teórica, mas vivencial, basada en la experiencia de las propias consumidoras, como una manera de la enfermería enterarse de la experiencia de conocer, manejar y divulgar la complejidad del fenómeno de las drogas, desde el contacto directo con el paciente, familia y comunidad, ya que este profesional es un miembro del equipo de salud que por la naturaleza de sus funciones y la cotidianidad de su trabajo se puede catalogar como agente de contacto continuo. La enfermería aborda los cuidados diarios a los pacientes de forma holística, por ello es fundamental su papel en la detección de problemas de salud y específicamente en problemas de adicción; son numerosas las ocasiones en las cuales las personas pueden expresar, a un enfermero o enfermera, su dependencia a una sustancia.

Este fenómeno lo han seguido con interés ${ }^{(6-8)}$ y han dejado ver la necesidad de su abordaje ya que el problema de las adolescentes en la calle que consumen drogas se relaciona con una marcada desvinculación tanto del hogar como de la educación formal, así como su factor de clase social pobre lo que las hace recurrir al consumo de alcohol y drogas ilícitas.

Podemos entonces ver que la adolescente sale de su casa como una manera de reivindicar el rol femenino, para demostrarle a la familia, a la sociedad y a su madre, como su mundo microsocial está lleno de maltrato y miserias, y lo expone así ante el mundo con su plasticidad e intuición femenina, diciéndonos que necesita ir a la calle para penetrar este mundo hostil, lleno de violencia callejera de todo tipo, a descubrir un delito que no haya arraigo, que llena vacíos, que produce orgasmos parciales, porque una sociedad mecánica, masculina y avasallante, penaliza desde muy adentro a estos seres femeninos que nutren, que cobijan, dan abrigo, alimentan y dan su humedad $y$ calidez a los hombres y mujeres del mañana para que el mundo siga adelante.

Tras haber expuesto el problema, vislumbrando que existen pocos registros de las situaciones "de" y "que" llevan a estas adolescentes a coger la calle y vivir en ella, nos preguntamos cuáles serán los puntos cardinales 
hacia donde se dirige esta investigación, y formulamos las siguientes preguntas:

¿Qué representa para las adolescentes de la calle el consumo de drogas?

¿Cómo percibe la adolescente de la calle el consumo de drogas en su grupo?

¿Qué cualidades atribuyen las adolescentes de la calle al consumo de droga y a sus relaciones con otros significativos?

Para dar respuestas a estas interrogantes y para alcanzar el propósito, nos planteamos el objetivo de revelar las circunstancias que rodean el consumo y el significado del consumo de drogas en las adolescentes de la calle de la ciudad de Valencia, Venezuela.

Considerando que en la actualidad varios estudios han abordado el consumo de drogas en poblaciones vulnerables ${ }^{(7,9-10)}$, pero pocos con adolescentes de sexo femenino en la calle, esperamos que este estudio puede contribuir para el conocimiento científico, para el desarrollo y formación de recursos humanos, para actuar con programas y acciones que resulten en la reducción de la demanda de drogas, conforme compromiso y políticas internacionales, y según lo establecido en las Américas $^{(1,11)}$.

\section{Metodología}

El estudio se abordó desde el paradigma cualitativo utilizando la Grounded Theory ${ }^{(12)}$ cuyo método de análisis es la comparación constante de los datos a través de la codificación abierta, axial y selectiva(12) y apoyándonos bajo las premisas del interaccionismo simbólico como herramientas para el acercamiento a las participantes ${ }^{(12)}$, previa evaluación, aprobación y autorización del comité de ética de la Escuela de Enfermería y del Centro Municipal de los Derechos del Niño y del Adolescentes del Estado Carabobo CEMDNA. A las adolescentes participantes menores de 18 años, se les proporcionó una declaración de consentimiento libre y esclarecido firmado por ellas. Se administró una entrevista semiestructurada a siete (7) adolescentes femeninas a quienes se les resguardo su identidad manteniéndolas en anonimato bajo pseudónimos de ciudades de Venezuela. Del análisis de los datos recogidos y la observación interpretativa en su contexto natural, se generaron partir de sus hallazgos significados y aproximaciones teóricas del mundo y de la realidad de la adolescente de la calle. Los criterios de selección de las informantes fueron: vivir en el Paseo Cabriales ubicado entre las Parroquias San José y San Blas del Municipio Valencia; participar voluntariamente en el estudio y con acuerdo firmado en la declaración de consentimiento libre y esclarecido para los adolescentes menores de 18 años; edades entre 15 y 19 años; haber consumido droga durante su permanencia en la calle; $y$, no encontrarse bajo los efectos de las drogas en el momento de la entrevista. Por petición de las informantes como medida individual para resguardar su identidad y su seguridad, las entrevistas se realizaron en el horario de 11:00pm a 3am, (1 hora y media - más o menos en algunos casos) en lugares seleccionados por ellas.

\section{Resultados}

Del análisis de los datos en bruto surgieron cinco categorías, las cuales comprendieron los temas para el análisis: a) La búsqueda de no ser la buena, b) Reafirmación del rol femenino, c) El consumo como experiencia, d) La mirada de los otros a la adolescente que consume drogas y e) La usuaria como cuidadora.

\section{Categoría A: En búsqueda de no ser la buena}

Las adolescentes entrevistadas dejan ver que el consumo de drogas es una manera de demostrarles a su madre y a sí misma, la necesidad de no seguir perpetuando el rol sumiso, abnegado, cumplidor de labores y tareas que la sociedad ha adjudicado a la mujer. La experiencia de consumir drogas viene a ser un elemento que rompe con este paradigma, es una manera de igualarse con sus pares masculinos, los que si pueden ser malos, sin límites, omnipotentes, creadores de reglas, ya que esto crea en la mujer un complejo de masculinidad (sentimientos de inferioridad, carácter vengativo y competencia con los hombres) que surgen de la dinámica familiar de la relación de la niña con los hombres de su familia(13) Dicho en palabras de las propias usuarias: ...Me fui para la calle por el vicio... Yo le decía si usted está brava yo me voy para la calle...(Miranda) ....Me fui para la calle cuando se murió mi mamá...Me gustó fue la vida de la calle...Si yo hacia algo malo ella me pegaba... (Zulia)... Inicie fumar cigarrillo desde los ocho años... y ....En la calle adquiere la labia, la firmeza, se vuelve hipócrita... (Apure). Significa que para las adolescentes consumidoras las drogas vienen a ser algo mas que un vicio, es la expresión de su posición ante la vida, rebelarse ante lo que le ocurre en casa y demostrar que al igual que los varones ellas también pueden hacer las cosas, que no existe diferencia sino estereotipos argumentados por la sociedad.

\section{Categoría B: Igualar el rol femenino}

Tiene que ver con la necesidad que muestran las adolescentes de igualar el papel cumplido por la mujer con el realizado por el hombre, es decir además de no 
querer mostrarse sumisas y "buenas", el consumo de drogas y la estancia en la calle vienen a hacer de la misma condición y naturaleza para un hombre y para una mujer, en este sentido Apure dice: ... la mujer es más peligrosa en la calle mil veces además Miranda agrega, ... Así como hay hombres buenos hay mujeres malas..., señalando además ...Más corren peligro los hombres que son sanos que andan buscando mujeres..., estas afirmaciones reivindican el papel fuerte de la mujer en un terreno que es común a ambos géneros, pero que de manera maníaca ellas lo interpretan como un triunfo para negar su dependencia, en un momento Zulia lo deja ver, el hombre se sabe defender mejor que una mujer... y ... Yo me fui a la calle desde que mi mama murió, con lo que la problemática de ser hombre o mujer en la cale y consumir drogas para sobrevivir en el mundo de la calle pasa a ser producto de un adolescente que constituye una representación social para sí mismo y para los otros en la sociedad, no es su condición biológica sino su condición social lo que justifica su comportamiento(14).

\section{Categoría C: El consumo como experiencia}

La complejidad del fenómeno humano al revelarse trata de hacerlo desde sus diversas perspectivas y es así como aparece la perspectiva biológica del consumo, así lo expresaron ellas, ... a uno la agarra la euforia, lo sube al cerebro así un sabroso, un cosquilleo así, los ojos volteados y sí claro se pone uno a ver...es como un escudo, no se una protección de pensar las cosas, los problemas. (Caracas) ...te hace retroceder la mente... ...te da ganas de tener sexo.... Te pone los ojos chinitos...y...Un choque eléctrico en la cabeza...Me avispo más que cuando estoy sana. (Zulia). ...Siento una euforia normal que altera el circuito nervioso, la droga no se va para ningún lado nada más para el cerebro...es estar activa...se puede escuchar cualquier de música... la gente quiere experimentar nuevas sensaciones con las drogas... (Miranda). Uno consume y olvida por el momento... (Apure). Este discurso viene a reivindicar lo planteado acerca de la implicación de las drogas en la sensación de recompensa que se cree es el mayor mediador de los efectos de las sustancias, inclinándose las adolescentes por una u otra droga de acuerdo a las necesidades de lograr un estado de ánimo que no puede conseguir por medios propios, las drogas despiertan sensaciones placenteras, inquietantes o pueden producir alucinaciones, y cada quien puede acercarse a ellas y consumirlas en diversas medidas en función de la necesidad sentida. Por otra parte el consumo de drogas es vivenciado como un hecho social y cultural, es decir el entorno social o cultural enseña a consumir sustancias como alternativa de diversión y enfrentamiento de dificultades.

\section{Categoría D: Los otros y su mirada a las adolescentes que consumen drogas}

Dentro del aspecto vincular, la mirada del otro resulta fundamental, es desde la percepción que tenemos de ésta podemos descubrir el grado de adaptación de las relaciones, ubicando lo adaptativo en relaciones óptimas, maduras, relativamente libres de distorsiones y gratificantes para las necesidades sexuales, agresivas y del yo adultas, para entrar en un intermedio constituido en relaciones caracterizadas por fijaciones tempranas, conflictos no resueltos y relaciones sadomasoquistas muy hostiles, por último lo más patológico sería la ausencia de relaciones con ninguna persona.

En la adolescencia, tal como lo plantea(15) el joven que está saliendo del cascarón, necesita desprenderse poco a poco de sus vínculos familiares infantiles y crear con sus padres una relación madura. Es como una necesidad convencerse de que se vale por sí mismo y demostrárselo a los demás. En esta especie de lucha, el enemigo más importante es él mismo y sobre todo la fuerte dependencia que tiene de sus padres, pero como esto no es fácil de reconocer, muchas veces el enemigo se traslada a la figura de los padres.

En las adolescentes entrevistadas vemos como al romper el límite del hogar y salir a la calle, la proyección que ha de depositar en sus padres, es puesta en el colectivo y allí deposita de manera defensiva su visión, así lo expresaron ella: ...la comunidad te rechaza porque consumes drogas. Las personas le tienen miedo a uno... algunos miran a uno como un animal...la gente piensa que uno es un desecho humano...aunque te estás riendo nadie sabe los que uno siente. (Zulia)....Me he sentido rechazada por la gente, muchas veces despreciada..., en este mundo la gente ve a uno como si fuera un extraterrestre, de otro mundo, de otro planeta... (Apure). Hoy en día la sociedad está más dañada...al drogadicto lo rechazan (Miranda). Esta forma de percibir la mirada del otro, confirma la idea de que el consumo de drogas tiene un aspecto de orden más defensivo y adaptativo que regresivo, por lo que abusar de una droga puede revertir estados regresivos mediante el refuerzo de defensas defectuosas del yo contra afectos poderosos tales como cólera, vergüenza y depresión, además de un Superyó cruel y ser excesivamente autocríticos. Sus comportamientos adictivos sirven para protegerse de una sensación de impotencia y desamparo mediante el control y la regulación de sus estados afectivos ${ }^{(16)}$

Las participantes dejan ver en su discurso la utilización masiva de defensas como la proyección, parecen situarse en una posición escindida (esquizoide), separadas de los otros, los cuales retornan para perseguir, 
atormentar, señalar ubicándose en lo paranoide, una posición que ubica en lo inmaduro, poco adaptativo, que por vía natural es vivenciado, pero de lo que hay que salir para crecer y desarrollarse; es el mismo punto central de establecer relaciones caracterizadas por el desapego, la sobre dependencia y aferramiento, en este caso a la droga y a la calle, que dejan ver una dificultad considerable para alcanzar un equilibrio cómodo entre acercarse y alejarse del otro, conservando la distancia por temor a salir lesionado por el rompimiento de una relación.

\section{Categoría E: La usuaria como cuidadora}

Los resultados aquí revelan que las adolescentes se refieren a la noción del consumo de drogas, no sólo como causante de enfermedad, de minusvalía, sino como reflejo de un hecho social, son las condiciones sociales las que hacen que emerjan este tipo de grupos, estos estilos de vida, estas formas de actuación, ante lo cual la adicta se posiciona, paradójicamente, en un rol de agente social protector, así lo expresaron ellas: Yo les digo a ellos que no sigan estos malos ejemplos... (Caracas). ... no yo no fumo delante de los niños, ellos son sagrados... yo le diría a una muchacha que no fumara drogas porque yo sé el trabajo que se pasa. (Zulia). ...le digo a la gente que se vea en este espejo...yo no voy a enviciar a otra persona para que se hunda... una persona que está empezando está a tiempo de salir. (Apure). ...le recomiendo a la gente que no consuma drogas...(Miranda).

\section{Discusión}

La búsqueda del significado del Consumo de Drogas para las Adolescentes de la Calle, en Valencia, Venezuela, surge como un primer intento para describir las acciones de un grupo de jóvenes de sexo femenino que viven en la calle, y cómo estas acciones las une a través de un símbolo: La Droga, en un primer momento al observar a estas adolescentes en su recorrido por la ciudad, uno piensa desde una posición funcionalista, que ha fallado algo en su familia, en la escuela en sí mismas, e incluso algunos pensaran en la necesidad de evitar el contacto entre estas adolescentes y "las adolescentes sanas".

Pero al conocerlas de cerca, escuchar sus palabras y observar parcialmente sus acciones, vemos que son el reflejo de una parcela de lo que hoy llamamos "juventud"; si ellas se encuentran haciendo cosas de jóvenes, no podemos explicar si es el terreno adecuado o no; lo que nos dejaron ver es su vulnerabilidad, su deseo de ser protegidas, sus fallas en los mecanismos de socialización, su miedo a desprenderse de las ideas familiares, así como lo intricado de su relación con la madre, un ser al cual adoran pero que sienten no haber tenido los suficientes recursos del Ser, del Sí Mismo, que les devolviera de manera elaborada cada una de sus dudas y necesidades.

Pareciera que antes de salir de sus casas, este grupo de adolescentes sintieron el vacío con que el grupo familiar y la misma sociedad se enfrentan a las inquietudes humanas, de allí que deciden ir a la calle como una manera de no seguir perpetuando el rol sumiso, abnegado, cumplidor de labores y tareas que la sociedad venezolana ha adjudicado a la mujer, esta experiencia de consumir drogas viene a ser un elemento que rompe con esta forma de ver el papel de la mujer $y$ así igualarse con sus pares masculinos.

Además la calle viene a representar el "estado oceánico", donde todo se puede, donde no hay límite, donde todo es dado, claro al llegar a ella, se dan cuenta de la realidad que les toca confrontar, sin embargo continúan en ella, porque sabemos lo difícil que para todos resulta declinar, más aun si se está en plena adolescencia o, se están haciendo cosas de jóvenes tal como lo señalan las entrevistadas ${ }^{(13)}$

Por otra parte la información obtenida nos permite visualizar la problemática de la drogas más allá de la mera recreación biológica. Iniciamos este articulo señalando "El consumo de drogas en el mundo es una conducta remota, ya que desde nuestros ancestros estas substancias han sido utilizadas, como medios para provocar alucinaciones y satisfacción, como formas de alejarse de la realidad y obtener placer"; en este momento podemos afirmar junto con las entrevistadas que el consumo de drogas es vivenciado como un hecho social y cultural, es decir el entorno social o cultural enseña a consumir sustancias como alternativa de diversión y enfrentamiento de dificultades, es así como la adicción sirve para protegerse de una sensación de impotencia y desamparo que es propia de cada adolescente.

Por último, paradójicamente en cada entrevistada vimos el surgimiento de su preocupación por el otro, del que "está sano", con invitaciones al no consumo, a evitar la calle, a no dejarse derrotar, posición resultante de la culpa que siente la persona ante lo social, sin darse cuenta que "su personaje" es el resultante de la necesidad que tiene el colectivo de espiar sus culpas, imperfecciones, asimetría, para no demostrar que con su poderío ha venido anulando al ser.

\section{Conclusión}

Al desconstruir las experiencias del consumo de las drogas en las adolescentes de la calle, podemos 
vislumbrar que esta experiencia se inicia como una manera de reafirmar su identidad a través del conflicto, tratando de no repetir lo visto en su hogar en su madre, como una manera de no ser buenas. Esta experiencia las lleva a reafirmar el rol trazado a la mujer en la sociedad ya que se consideran vulnerables, carentes de una fuerza física pero sobre todo social que les permita ejercer roles al igual que los hombres. Esto las Ileva a centrar su consumo en la experiencia física dejando a un lado sensaciones, sentimientos, ansiedades $y$ conflictos no resueltos, como parte de la prevalencia de un pensamiento mágico que las lleve a salir o afrontar su vivencia traumática sin dolor. El consumo es visto con rechazo por los otros, estereotipándolas, segregándolas y estigmatizándolas, con lo que se reafirma el papel secundario de la mujer, no obstante en ellas vemos la fuerza intrínseca a lo femenino que cuida, que acciona y que se inmola por el otro, como una manera de cuidar.

Creemos que los resultados de este estudio contribuyen con el conocimiento de la enfermería y otras disciplinas, con miras a obtener una mejor comprensión del consumo de drogas por las adolescentes de sexo femenino que viven en la calle, colaborando para establecer nuevas formas de atender a esta población.

\section{Agradecimientos}

Agradecemos a la Comisión Interamericana para el Control del Abuso de Drogas/CICAD de la Secretaria de Seguridad Multidimensional/SSM de la Organización de Ios Estados Americanos/OEA, la Secretaria Nacional de Políticas sobre Drogas/SENAD do Gabinete de Seguridad Institucional/Brasil, la Escuela de Enfermería de Ribeirao Preto de la Universidad de Sao Paulo y Centro Colaborador de la Organización Mundial de la Salud para el Desarrollo de la Investigación en Enfermería, la población representada en los estudios de investigación, bien como a las autoridades de las universidades representadas por los participantes del Programa En-Line de Especialización en Investigación sobre el Fenómeno de las Drogas, periodos 2006, 2007, 2008 y 2009.

\section{Referencias}

1. Wright MGM, Gliksman L, Khenti A, Furegato ARF. Investigación sobre el fenómeno de las drogas bajo el abordaje de los estudios multicéntricos en América Latina y Caribe. Rev. Latino-Am. Enfermagem. 2009;17(spe):759-61.

2. United Nations Office on Drugs and Crime. World drug report 2007: analysis. United Nations Publication, 2007. [access Oct 09 2007]. Available from: http://www. unodc.org/documents/about-unodc/AR06_fullreport.pdf 3. Oficina Nacional Antidrogas. Estatísticas del consumo de drogas en centros de tratamiento. Venezuela, 2006. [acceso 9 Oct 2007]. Disponible en: www.ona.gob.ve Organización Mundial de la Salud. Programación para la salud y el desarrollo de los adolescentes. Genebra: OMS; 1999. Serie de informes técnicos, 886.

5. Oliveira M. A rua: um acolhimento falaz as crianças que nela vivem. Rev. Latino-Am. Enfermagem. setembrooutubro 2003;11(5):622-9.

6. Mendosa N, Henríquez E, Frari S. El Consumo de Alcohol y la Violencia Desde la Perspectiva de las Adolescentes. Relatorio Final. Washington: Proyecto CICAD-OEA; 2003.

7. Facundo FRG, Pedrão LJ. Personal and interpersonal risk factors in the consumption of illicit drugs by marginal adolescents and young people from juvenile gangs. Rev. Latino-Am. Enfermagem. junho 2008;16(3):368-74.

8. Horta RL, Horta BL, Pinheiro RT, Morales B, Strey MN. Tabaco, álcool e outras drogas entre adolescentes em Pelotas, Rio Grande do Sul, Brasil: uma perspectiva de gênero. Cad Saúde Pública abril 2007;23(4):775-83.

9. Penna LHG, Carinhanha Joana I, Leite LC. The educative practice of professional caregivers at shelters: coping with violence lived by female adolescents. Rev. Latino-Am. Enfermagem. dez 2009;17(6):981-7.

10. Oliveira JF, Paiva MS, Valente CML. The interference of the care context with the visibility of the drug consumption by women. Rev. Latino-Am. Enfermagem. Abr 2007;15(2):247-52.

11. Mendes IAC, Marziale MHP. Developing competencies for drug demand reduction. Rev. Latino-Am. Enfermagem. ago 2008;16(spe):505-6.

12. Strauss A, Corbin J. Basics of qualitative research: techniques and procedures for developing grounded theory. 2nd. ed. London (UK): Saje; 1998. p. 136-267.

13. Blumer H. Symbolic Interaccionismo; perspective end method. Berkeley: University of California; 1969.

14. Groppo LA. Juventude: ensaios sobre sociologia e história das juventudes modernas. Rio de Janeiro (RJ): DIFEL; 2000. p. 19-23.

15. Vallejo Nájera JA. Guía Práctica de Psicología. Madri: Temas de Hoy; 2005.

16. Gabbard GO. Psiquiatría y Psicodinamica en la práctica Clínica. 3th. ed. Madri: Medica Panamericana; 2002. 